WCH-326

Rev. 0

River Corridor Closure Contract

\title{
100-K Target Analyte List Development for Soil
}

April 2010

For Public Release

Washington Closure Hanford

Prepared for the U.S. Department of Energy, Richland Operations Office Office of Assistant Manager for River Corridor 
TRADEMARK DISCLAIMER

Reference herein to any specific commercial product, process, or service by trade name, trademark, manufacturer, or otherwise, does not necessarily constitute or imply its endorsement, recommendation, or favoring by the United States Government or any agency thereof or its contractors or subcontractors.

This report has been reproduced from the best available copy.

Printed in the United States of America 


\section{DOCUMENT COMTROL

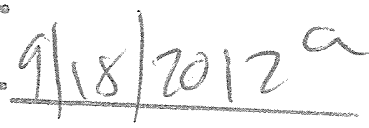 a \\ WCH-326 \\ Rev. 0}

\section{STANDARD APPROVAL PAGE}

Title:

100-K Target Analyte List Development for Soil

Author Name: R. W. Ovink, Integration Project Task Lead

Approval: $\quad$ J. A. Lerch, Mission Completion

Signature


WCH-326

Rev. 0

\section{River Corridor Closure Contract}

\section{0-K Target Analyte List Development for Soil}

April 2010

Author:

R. W. Ovink

For Public Release 


\section{TABLE OF CONTENTS}

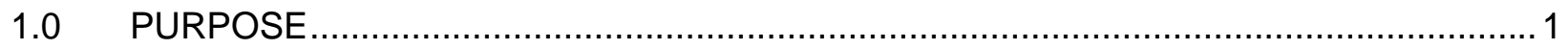

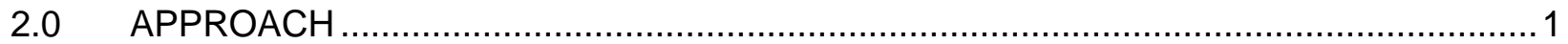

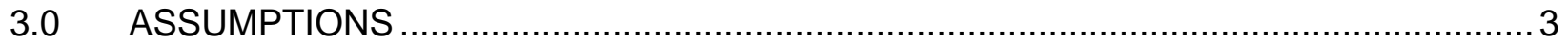

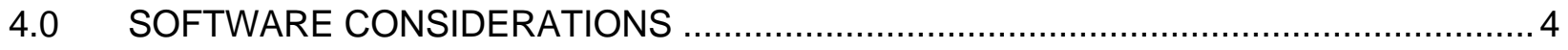

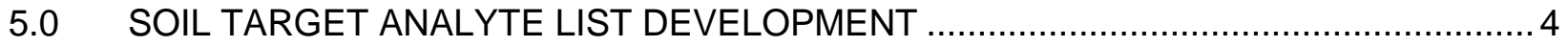

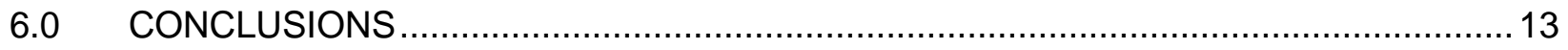

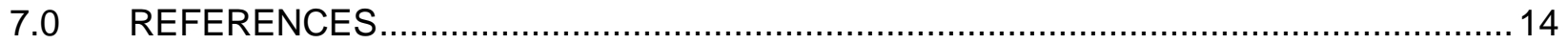

\section{TABLES}

1. Documents Used to Develop the 100-K Decision Unit Analyte List. ........................... 4

2. Summary of $100-\mathrm{K}$ Initial Target Analytes and References. ..................................... 6

3. $\quad 100-K$ Soil Analytes Excluded from Further Consideration. ...................................... 8

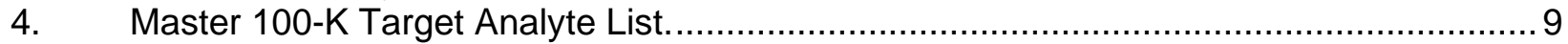

5. 100-K Location-Specific Target Analyte List. ................................................. 12

\section{APPENDIX}

A. $\quad$ 100-K TARGET ANALYTE MEASLES CHART 


\subsection{PURPOSE}

This report documents the process used to identify source area target analytes in support of the 100-K Area remedial investigation/feasibility study (RI/FS) addendum to the Integrated 100 Area Remedial Investigation/Feasibility Study Work Plan (DOE/RL-2008-46, Rev. 0). A "target analyte" is defined as a constituent suspected of being site-related that is carried into an investigation plan for characterization through sampling and analysis by approved laboratory methods. Target analytes identified for the 100 and 300 Areas must support RI/FS nature and extent characterization plus final remedial action decisions for source areas. This report also establishes the analyte exclusion criteria applicable for the 100 and 300 Area use and the analytical methods needed to analyze the refined target analytes.

\subsection{APPROACH}

The approach for the development of vadose zone soil target analytes is a multi-step process. In Steps 1 and 2, initial and master analytes lists were developed. The third step developed location-specific target analyte lists for waste sites where additional characterization is planned. Finally, the analyte list will receive regulatory review and input. This will result in final location-specific target analytes.

\section{Step 1 - Prepare Initial Master Target Analyte}

Characterization data for vadose zone soils are not available for addressing uncertainties associated with the nature and extent of contamination in the vadose zone. Therefore, remediation and characterization information (historic and current) is identified and reviewed to develop an initial list of target analytes to represent potential contamination in the vadose zone. The following types of reference documents and information sources are evaluated:

- Focused feasibility studies (FFS), limited field investigation (LFI) reports

- Interim action records of decision (IARODs)

- Cleanup verification documents (cleanup verification packages [CVPs], remaining sites verification packages [RSVPs])

- Technical baseline reports

- Databases containing analytical data resulting from these activities (i.e., characterization, remediation, waste management information)

- Other pertinent documents 


\section{Step 2 - Prepare Master Target Analyte List}

After the initial target analyte list is compiled, the information undergoes additional review steps to remove analytes using generally-accepted exclusion criteria; a comparison of the soil target analyte list to the groundwater contaminants of potential concern (COPC) list is conducted, and the appropriate analytical methods and detection limits for the master target analyte list is identified.

At the conclusion of this step, the master target analyte list is established. The master target analyte list is comprehensive and includes all the analytes that have the potential to be present in the vadose zone and are important for waste site remediation within the area. The following steps are taken to prepare the master target analyte list.

- Apply the following generally accepted exclusion criteria to the initial set of target analytes.

- Radionuclides with half-lives less than 3 years (and no significant "daughters")

- Naturally occurring radionuclides associated with background radiation (e.g., K-40, Th-230, Th-232, and Ra-226)

- Essential nutrients (minerals)

- Analytes that have no toxicity values (based on the hierarchy of toxicity values recommended by the U.S. Environmental Protection Agency (EPA) in Human Health Toxicity Values for Superfund Risk Assessments [OSWER Directive 9285.7-53]).

- Compare the master target analyte list for vadose zone soil with the groundwater COPC list developed for the area. Groundwater COPCs not found on the master target analyte list are further evaluated to determine if there is a valid basis for their inclusion.

- Identify appropriate analytical methods for each analyte on the master target analyte list. Determine if the detection limits for each target analyte can achieve the remedial action goals for direct exposure, groundwater protection, and Columbia River protection.

\section{Step 3 - Develop Location-Specific Target Analyte List}

The master target analyte list represents all potential target analytes that could be present in the vadose zone for the waste site area. Location-specific target analytes will be identified from the master list using the following approach.

- Identify the contaminants of concern (COCs) for the specific waste sites where characterization is proposed from the applicable IAROD (which reflects information from LFI and technical baseline reports). If the characterization location is not at a waste site, evaluate information from waste sites in the vicinity (where available), and include these analytes on the location-specific target analyte list.

- Identify the COCs for the specific waste site locations from the verification documentation (CVPs or RSVPS). If the characterization location is not at a waste site, evaluate information 
from waste sites in the vicinity (where available), and include these analytes on the locationspecific target analyte list.

- Evaluate local groundwater monitoring well data (wells located within waste site "zones of influence"). Determine if groundwater COPCs have been analyzed for in these local wells.

- If the groundwater COPCs have been analyzed for but not detected, then these analytes will not be included on the location-specific target analyte list.

- If the groundwater COPCs have been analyzed for and have been detected, then these analytes will be included on the location-specific target analyte list.

- If the groundwater COPCs have not been analyzed for, then an additional evaluation will be performed to determine if there is a data need. If there is a data need, these COPCs will be included on the waste-site specific target analyte list.

\section{Step 4 - Agency Review of Locations and Location-Specific Target Analyte Lists}

Following development of the location-specific target analyte list pursuant to Steps 1 through 3 above, the agencies will review the locations and the location-specific target analyte lists to determine whether adjustments/modifications are required to address information needs for the area. This review is intended to provide an opportunity to address any information requirements not identified in Steps 1 through 3. When additional information needs are identified, the agencies will modify the locations for additional characterization or the location-specific target analyte lists to reflect the additions/modifications needed for the area.

\subsection{ASSUMPTIONS}

- Historical resources (e.g., LFI, qualitative risk assessment, and CVP/RSVP documents) contain contaminant lists that are comprehensive with respect to characterizing environmental impacts from the 100 and 300 Area Hanford Site operations.

- Older analytical data (e.g., pre-Comprehensive Environmental Response, Compensation, and Liability Act of 1980 [CERCLA]) reflect laboratory state-of-the-art procedures. Analytical methods have improved, resulting in lower detection limits for many analytes and better data quality assurance/quality control.

- Characterization activities implemented since initiating remediation under the IARODs may provide additional contaminant information that should be considered during pending RI/FS work processes.

- Post-remediation characterization and cleanup verification data reflect focused lists of analytes that are unique to each waste site and have been evaluated against IAROD cleanup requirements.

- Examining existing data and waste site process information will be useful in developing laboratory analytical needs for RI/FS characterization tasks. 
- Universally accepted exclusion criteria may be applied to the initial target analyte list to develop a "refined" list.

- Additional exclusion criteria (e.g., statistical Hanford Site background comparisons, infrequently detected analytes, and analytes not detected at concentrations/activities exceeding required cleanup levels) may be applied during the RI/FS process as more data become available.

\subsection{SOFTWARE CONSIDERATIONS}

No statistical or algebraic calculations are performed for this activity. The evaluations conducted included analyte comparisons/sorting using Microsoft ${ }^{\circledast}$ Excel $^{\circledast}$.

\subsection{SOIL TARGET ANALYTE LIST DEVELOPMENT}

\section{Master Target Analyte Identification}

1. The documents listed in Table 1 were used to develop the 100-K target analyte list.

Table 1. Documents Used to Develop the 100-K Decision Unit Analyte List. (3 Pages)

\begin{tabular}{|c|c|c|}
\hline Reference & Document Number & Document Type \\
\hline $\begin{array}{l}\text { 1. Cleanup Status Report for the } 116-K E-1 \text { and } \\
116-K W-1 \text { Cribs }\end{array}$ & BHI-01737 & $\mathrm{BHI}$ report \\
\hline $\begin{array}{l}\text { 2. Cleanup Verification Package for the } 100-K-55: 1 \text { and } \\
\text { 100-K-56:1 Pipelines and the } 116-K W-4 \text { and } \\
\text { 116-KE-5 Heat Recovery Stations }\end{array}$ & CVP-2005-00006 & CVP \\
\hline $\begin{array}{l}\text { 3. Cleanup Verification Package for the 116-KE-4 } \\
\text { Retention Basin }\end{array}$ & CVP-2005-00002 & CVP \\
\hline $\begin{array}{l}\text { 4. Cleanup Verification Package for the } 116-\mathrm{KW}-3 \\
\text { Retention Basin }\end{array}$ & CVP-2004-00001 & CVP \\
\hline 5. Cleanup Verification Package for the $116-\mathrm{K}-1$ Crib & CVP-2003-00024 & CVP \\
\hline $\begin{array}{l}\text { 6. Cleanup Verification Package for the 116-K-2 Effluent } \\
\text { Trench }\end{array}$ & CVP-2006-00001 & CVP \\
\hline
\end{tabular}

\footnotetext{
${ }^{\circledR}$ Microsoft and Excel are registered trademarks of Microsoft Corporation in the United States and/or other countries.
} 
Table 1. Documents Used to Develop the 100-K Decision Unit Analyte List. (3 Pages)

\begin{tabular}{|c|c|c|}
\hline Reference & Document Number & Document Type \\
\hline $\begin{array}{l}\text { 7. Remaining Sites Verification Package for } 128-\mathrm{K}-1 \text {, } \\
\text { 100-K Burning Pit, Attachment to Waste Site } \\
\text { Reclassification Form 2004-042 }\end{array}$ & WSRF 2004-042 & RSVP \\
\hline $\begin{array}{l}\text { 8. Remaining Sites Verification Package for } 100-\mathrm{K}-29 \text {, } \\
\text { 183-KE Sandblasting Site, Attachment to Waste Site } \\
\text { Reclassification Form 2004-040 }\end{array}$ & WSRF 2004-040 & RSVP \\
\hline $\begin{array}{l}\text { 9. Remaining Sites Verification Package for } 100-\mathrm{K}-30 \text {, } \\
\text { 183-KE Sulfuric Acid Tank (West Tank), Attachment to } \\
\text { Waste Site Reclassification Form 2003-036 }\end{array}$ & WSRF 2003-036 & RSVP \\
\hline $\begin{array}{l}\text { 10. Remaining Sites Verification Package for } 100-K-31 \text {, } \\
\text { 183-KE Sulfuric Acid Tank (East Tank), Attachment to } \\
\text { Waste Site Reclassification Form 2004-038 }\end{array}$ & WSRF 2004-038 & RSVP \\
\hline $\begin{array}{l}\text { 11. Remaining Sites Verification Package for 100-K-32, } \\
\text { 183-KW Sulfuric Acid Tank Bases (East Tank), } \\
\text { Attachment to Waste Site Reclassification Form } \\
\text { 2004-039 }\end{array}$ & WSRF 2004-039 & RSVP \\
\hline $\begin{array}{l}\text { 12. Remaining Sites Verification Package for 100-K-33, } \\
\text { 183-KW Sulfuric Acid Tank Bases (West Tank), } \\
\text { Attachment to Waste Site Reclassification Form } \\
\text { 2004-041 }\end{array}$ & WSRF 2004-041 & RSVP \\
\hline $\begin{array}{l}\text { 13. } 100 \text { Area Source Operable Unit Focused Feasibility } \\
\text { Study Report }\end{array}$ & DOE/RL-94-61 & FFS \\
\hline $\begin{array}{l}\text { 14. Sampling and Analysis Plan for Investigating } \\
\text { Chromium Groundwater Contamination Near the } \\
\text { 105-KW Reactor }\end{array}$ & DOE/RL-2008-33 & SAP \\
\hline $\begin{array}{l}\text { 15. Limited Field Investigation Report for the } 100-K R-1 \\
\text { Operable Unit }\end{array}$ & DOE/RL-93-78 & LFI \\
\hline 16. Radiological Characterization of the Retired 100 Areas & $\begin{array}{l}\text { UNI-946 } \\
\text { Dorian, J. J. and V. R. } \\
\text { Richards }\end{array}$ & UNI report \\
\hline $\begin{array}{l}\text { 17. Record of Decision for the 100-KR-2 Operable Unit, } \\
\text { Hanford Site }\end{array}$ & EPA/ROD/R10-99/059 & ROD \\
\hline $\begin{array}{l}\text { 18. Interim Action Record of Decision for the 100-BC-1, } \\
\text { 100-BC-2, 100-DR-1, 100-DR-2, 100-FR-1, 100-FR-2, } \\
\text { 100-HR-1, 100-HR-2, 100-KR-1, 100-KR-2, 100-IU-2, } \\
\text { 100-IU-6, and 200-CW-3 Operable Units, Hanford Site } \\
\text { (Remaining Sites ROD) }\end{array}$ & EPA/ROD/R10-99/039 & IAROD \\
\hline $\begin{array}{l}\text { 19. Record of Decision for the 100-BC-1, 100-BC-2, } \\
\text { 100-DR-1, 100-DR-2, 100-FR-2, 100-HR-2, and } \\
\text { 100-KR-2 Operable Units, Hanford Site (Burial } \\
\text { Grounds ROD) }\end{array}$ & EPA/ROD/R10-00/121 & ROD \\
\hline
\end{tabular}


Table 1. Documents Used to Develop the 100-K Decision Unit Analyte List. (3 Pages)

\begin{tabular}{|c|c|c|}
\hline Reference & Document Number & Document Type \\
\hline $\begin{array}{l}\text { 20. Dangerous Waste Portion of the Resource } \\
\text { Conservation and Recovery Act Permit for the } \\
\text { Treatment, Storage, and Disposal of Dangerous } \\
\text { Waste, Permit No. WA7 } 890008967 \text {, Closure Unit 16, } \\
\text { 1706-KE Waste Treatment System }\end{array}$ & WA7 890008967 & $\begin{array}{l}\text { Dangerous } \\
\text { waste permit } \\
\text { application }\end{array}$ \\
\hline $\begin{array}{ll}\text { BHI } & =\text { Bechtel Hanford, Inc. } \\
\text { CVP } & =\text { cleanup verification package } \\
\text { DOE/RL } & =\text { U.S. Department of Energy, Richland Operations } \\
\text { Office } & \\
\text { EPA } & =\text { U. S. Environmental Protection Agency } \\
\text { FFS } & =\text { focused feasibility study }\end{array}$ & $\begin{array}{ll}\text { LFI } & =\text { limited field inv } \\
\text { ROD } & =\text { record of decis } \\
\text { RSVP } & =\text { remaining sites } \\
\text { SAP } & =\text { sampling and a } \\
\text { UNI } & =\text { United Nuclear } \\
\text { WSRF } & =\text { waste site recla }\end{array}$ & $\begin{array}{l}\text { gation } \\
\text { rification package } \\
\text { ysis plan } \\
\text { lustries } \\
\text { fication form }\end{array}$ \\
\hline
\end{tabular}

2. The initial list of target analytes presented in Table 2 was created from the review and evaluation of the Table 1 documents.

Table 2. Summary of 100-K Initial Target Analytes and References. (2 Pages)

\begin{tabular}{|c|c|c|c|}
\hline Analyte & Reference & Analyte & Reference \\
\hline \multicolumn{4}{|c|}{ Radionuclides } \\
\hline 1. Americium-241 & DOE/RL-94-61 & 13. Potassium-40 & WSRF 2004-042 \\
\hline 2. Carbon-14 & DOE/RL-94-61 & 14. Radium-226 & WSRF 2004-042 \\
\hline 3. Cesium-134 & DOE/RL-94-61 & 15. Sodium-22 & DOE/RL-94-61 \\
\hline 4. Cesium-137 & CVP-2003-00024 & 16. Strontium-90 & CVP-2003-00024 \\
\hline 5. Cobalt- 60 & CVP-2003-00024 & 17. Technetium-99 & DOE/RL-2008-33 \\
\hline 6. Gross beta & DOE/RL-93-78 & 18. Thorium-228 & WSRF 2004-042 \\
\hline 7. Europium-152 & CVP-2005-0006 & 19. Thorium-232 & DOE/RL-94-61 \\
\hline 8. Europium-154 & CVP-2005-0006 & 20. Tritium & DOE/RL-2008-33 \\
\hline 9. Europium-155 & DOE/RL-94-61 & 21. Uranium-233/234 & CVP-2004-00001 \\
\hline 10. Nickel-63 & CVP-2006-00001 & 22. Uranium-235 & CVP-2004-00001 \\
\hline 11. Plutonium-238 & DOE/RL-94-61 & Uranium_220 & 只 \\
\hline 12. Plutonium-239/240 & CVP-2004-00001 & 23. Ularmum- 30 & DUE/RL-2U00-3J \\
\hline \multicolumn{4}{|c|}{ Nonradionuclides } \\
\hline 1. 1, 1-Dichloroethene & HEIS/Groundwater & 33. Dibenz[a,h]anthracene & WSRF 2004-042 \\
\hline 2. Acenaphthene & DOE/RL-93-78 & 34. Di-n-butylphthalate & DOE/RL-93-78 \\
\hline 3. Aluminum & DOE/RL-93-78 & 35. Ethylene glycol & CVP-2005-00006 \\
\hline 4. Anthracene & WSRF 2004-042 & 36. Fluoranthene & WSRF 2004-042 \\
\hline 5. Antimony & DOE/RL-94-61 & 37. Fluoride & DOE/RL-93-78 \\
\hline 6. Aroclor-1016 & WSRF 2004-04 & $\begin{array}{l}\text { 38. Indeno(1,2,3- } \\
\text { cd)pyrene }\end{array}$ & WSRF 2004-042 \\
\hline 7. Aroclor-1221 & WSRF 2004-04 & 39. Iron & DOE/RL-93-78 \\
\hline 8. Aroclor-1232 & WSRF 2004-04 & 40. Lead & CVP-2005-00006 \\
\hline 9. Aroclor-1242 & WSRF 2004-04 & 41. Magnesium & DOE/RL-93-78 \\
\hline 10. Aroclor-1248 & WSRF 2004-04 & 42. Manganese & DOE/RL-94-61 \\
\hline 11. Aroclor-1254 & WSRF 2004-04 & 43. Mercury & CVP-2005-00006 \\
\hline 12. Aroclor-1260 & WSRF 2004-04 & 44. Methylene chloride & DOE/RL-93-78 \\
\hline 13. Arsenic & CVP-2005-0006 & 45. Nickel & DOE/RL-93-78 \\
\hline 14. Barium & CVP-2005-0006 & 46. Nitrate-nitrite & DOE/RL-93-78 \\
\hline
\end{tabular}


Table 2. Summary of 100-K Initial Target Analytes and References. (2 Pages)

\begin{tabular}{|c|c|c|c|}
\hline Analyte & Reference & Analyte & Reference \\
\hline 15. Benzene (GW COPC) & HEIS/Groundwater & 47. Pentachlorophenol & DOE/RL-94-61 \\
\hline 16. Benzo(a)anthracene & WSRF 2004-042 & 48. Phenanthrene & WSRF 2004-042 \\
\hline 17. Benzo(a)pyrene & WSRF 2004-042 & 49. Phosphate & DOE/RL-93-78 \\
\hline 18. Benzo(b)fluoranthene & WSRF 2004-042 & 50. Potassium & DOE/RL-93-78 \\
\hline 19. Benzo(ghi)perylene & WSRF 2004-042 & 51. Pyrene & WSRF 2004-042 \\
\hline 20. Benzo(k)fluoranthene & WSRF 2004-042 & 52. Selenium & WSRF 2004-042 \\
\hline 21. Beryllium & DOE/RL-93-78 & 53. Silver & WSRF 2004-042 \\
\hline $\begin{array}{l}\text { 22. Bis(2-ethylhexyl) } \\
\text { phthalate }\end{array}$ & WSRF 2004-042 & 54. Sodium & DOE/RL-93-78 \\
\hline 23. Cadmium & CVP-2005-00006 & 55. Sulfate & WSRF 2003-036 \\
\hline 24. Calcium & DOE/RL-93-78 & 56. Tetrachloroethene & DOE/RL-93-78 \\
\hline 25. Carbazole & WSRF 2004-042 & 57. Thallium & DOE/RL-93-78 \\
\hline $\begin{array}{l}\text { 26. Carbon tetrachloride } \\
\text { (GW COPC) }\end{array}$ & HEIS/Groundwater & uene & DOE/RL-93-78 \\
\hline $\begin{array}{l}\text { 27. Chloroform } \\
\text { (GW COPC) }\end{array}$ & HEIS/Groundwater & $\begin{array}{l}\text { 59. Total petroleum } \\
\text { hydrocarbons }\end{array}$ & WSRF 2004-042 \\
\hline $\begin{array}{l}\text { 28. Chromium } \\
\text { (hexavalent) }\end{array}$ & CVP-2003-00024 & 60. Trichloroethene & DOE/RL-2008-33 \\
\hline 29. Chromium (total) & CVP-2003-00024 & 61. Vanadium & DOE/RL-93-78 \\
\hline 30. Chrysene & WSRF 2004-042 & \multirow{3}{*}{ 62. Zinc } & \multirow{3}{*}{ DOE/RL-94-61 } \\
\hline 31. Cobalt & DOE/RL-93-78 & & \\
\hline 32. Copper & DOE/RL-93-78 & & \\
\hline
\end{tabular}

NOTE: The primary references are listed for each analyte; most analytes were referenced in multiple documents.

CVP = cleanup verification package

$\mathrm{DOE} / \mathrm{RL} \quad=$ U.S. Department of Energy, Richland Operations Office

GW COPC = groundwater contaminant of potential concern

HEIS = Hanford Environmental Information System

WSRF = waste site reclassification form

3. The generally accepted exclusion criteria that follow were applied to the initial soil target analyte list to identify the excluded analytes listed in Table 3 and to develop the refined target analyte list presented in Table 4.

- Radionuclides with half-lives less than 3 years (and no significant "daughters")

- Naturally occurring radionuclides associated with background radiation

- Essential nutrients (minerals)

- Analytes that have no toxicity values (per the most-current Cleanup Levels and Risk Calculations Database [CLARC] Table). 
Table 3. 100-K Soil Analytes Excluded from Further Consideration.

\begin{tabular}{|c|c|c|c|}
\hline Analyte & Exclusion Rationale & Daughters & Half-Life \\
\hline \multicolumn{4}{|c|}{ Radionuclides } \\
\hline Cerium-144 & Half-life less than 3 years (284.6d) & $\begin{array}{l}\text { Pr-144m (1.2m), } \\
\text { Pr-144 (17.28m), } \\
\text { and Nd-144 } \\
\text { (stable) }\end{array}$ & NA \\
\hline Cesium-134 & Half-life less than 3 years (2.065y) & Ba-134 (stable) & NA \\
\hline Cobalt-58 & Half-life less than 3 years (70.88d) & Ni-58 (stable) & $\mathrm{NA}$ \\
\hline Iron-59 & Half-life less than 3 years (44.51d) & Co-59 (stable) & NA \\
\hline Manganese-54 & Half-life less than 3 years $(612.2 d)$ & Fe-54 (stable) & NA \\
\hline Ruthenium-103 & Half-life less than 3 years $(39.27 d)$ & $\begin{array}{l}\text { Rh-103m (56.12m) } \\
\text { and Rh-103 } \\
\text { (stable) }\end{array}$ & NA \\
\hline Ruthenium-106 & Half-life less than 3 years (1.020y) & $\begin{array}{l}\text { Rh-106 (29.9s) and } \\
\text { Pd-106 (stable) }\end{array}$ & NA \\
\hline Sodium-22 & Half-life less than 3 years (2.605y) & Ne-22 (stable) & NA \\
\hline Tin-113 & Half-life less than 3 years (115.1d) & $\begin{array}{l}\text { In-113m (1.658h) } \\
\text { and In-113 (stable) }\end{array}$ & NA \\
\hline Actinium-228 & $\begin{array}{l}\text { Decay daughter (Th-232/Ra-228); in } \\
\text { equilibrium with parent }\end{array}$ & NA & 6.15 hours \\
\hline Lead-212 & $\begin{array}{l}\text { Decay daughter (Th-232/Ra-228); in } \\
\text { equilibrium with parent }\end{array}$ & NA & 10.6 hours \\
\hline Lead-214 & $\begin{array}{l}\text { Decay daughter (Ra-226); in equilibrium } \\
\text { with parent }\end{array}$ & NA & 26.8 minutes \\
\hline Potassium-40 & Naturally occurring background radiation & NA & $1.28 \mathrm{E} 9$ years \\
\hline Radium-224 & $\begin{array}{l}\text { Decay daughter (Th-232/Ra-228); in } \\
\text { equilibrium with parent }\end{array}$ & NA & 3.66 days \\
\hline Radium-226 & $\begin{array}{l}\text { Only potential source from naturally- } \\
\text { occurring background radiation (insufficient } \\
\text { in-growth time for Hanford introduced } U \text { as } \\
\text { decay daughter of U-234/Th-230) }\end{array}$ & NA & 1.6 E3 years \\
\hline Radium-228 & $\begin{array}{l}\text { Decay daughter (Th-232); in equilibrium } \\
\text { with parent }\end{array}$ & NA & 5.76 years \\
\hline Thorium-228 & $\begin{array}{l}\text { Decay daughter (Th-232/Ra-228); in } \\
\text { equilibrium with parent }\end{array}$ & NA & 1.91 years \\
\hline Thorium-230 & $\begin{array}{l}\text { Only potential source from naturally- } \\
\text { occurring background radiation (insufficient } \\
\text { in-growth time for Hanford introduced } U \text { as } \\
\text { decay daughter of } U-234 \text { ) }\end{array}$ & NA & 7.7 E4 years \\
\hline Thorium-232 & Naturally occurring background radiation & NA & 1.4 E10 years \\
\hline Thorium-234 & $\begin{array}{l}\text { Decay daughter of U-238; in equilibrium } \\
\text { with parent }\end{array}$ & NA & 2.41 days \\
\hline \multicolumn{4}{|c|}{ Nonradionuclides } \\
\hline Calcium & Essential nutrient & NA & NA \\
\hline Iron & Essential nutrient & NA & NA \\
\hline Magnesium & Essential nutrient & NA & NA \\
\hline Phosphate & Essential nutrient & NA & NA \\
\hline Potassium & Essential nutrient & NA & NA \\
\hline Sodium & Essential nutrient & NA & NA \\
\hline Sulfate & No soil toxicity information available & NA & NA \\
\hline
\end{tabular}

NA = not applicable 
4. This step reconciles the refined soil target analytes with the groundwater COPCs developed for the area. Groundwater COPCs not found on the refined soils list are further evaluated. The default action is to include all groundwater COPCs on the refined soil target analyte list, unless there is a valid basis for their exclusion. The analytes added to Table 4 that are groundwater COPCs are presented in italics.

5. The appropriate analytical methods for the refined target analytes, taking into account action levels and detection limits, are presented in Table 4.

Table 4. Master 100-K Target Analyte List. (3 Pages)

\begin{tabular}{|c|c|c|c|c|c|}
\hline \multirow[b]{2}{*}{ Target Analyte } & \multirow{2}{*}{$\begin{array}{c}\text { Practical } \\
\text { Quantitation } \\
\text { Limits }^{\mathrm{a}}\end{array}$} & \multicolumn{3}{|c|}{ Preliminary Cleanup Goals ${ }^{\mathrm{a}, \mathrm{b}}$} & \multirow[b]{2}{*}{ Analytical Methods } \\
\hline & & $\begin{array}{c}\text { Direct } \\
\text { Exposure }\end{array}$ & $\begin{array}{l}\text { Groundwater } \\
\text { Protection }\end{array}$ & $\begin{array}{c}\text { River } \\
\text { Protection }\end{array}$ & \\
\hline \multicolumn{6}{|c|}{ Radionuclides } \\
\hline 1. Cesium-137 & 0.1 & 6.2 & $\mathrm{NV}^{\mathrm{c}}$ & $\mathrm{NV}^{\mathrm{c}}$ & \multirow{6}{*}{$\begin{array}{l}\text { 1. Gamma energy } \\
\text { analysis }\end{array}$} \\
\hline 2. Cobalt- 60 & 0.05 & 1.4 & $N V^{c}$ & $N V^{c}$ & \\
\hline 3. Europium-152 & 0.1 & 3.3 & $\mathrm{NV}^{\mathrm{C}}$ & $\mathrm{NV}^{\mathrm{C}}$ & \\
\hline 4. Europium-154 & 0.1 & 3.0 & $N V^{c}$ & $N V^{c}$ & \\
\hline 5. Europium-155 & 0.1 & 125 & $\mathrm{NV}^{\mathrm{c}}$ & $\mathrm{NV}^{\mathrm{c}}$ & \\
\hline 6. Americium-241 & 1 & 31.1 & $\mathrm{NV}^{\mathrm{C}}$ & $\mathrm{NV}^{\mathrm{C}}$ & \\
\hline 7. Plutonium-238 & 1 & 37.4 & $\mathrm{NV}^{\mathrm{C}}$ & $\mathrm{NV}^{\mathrm{C}}$ & \multirow{2}{*}{$\begin{array}{ll}\text { 2. } & \begin{array}{l}\text { Isotopic- } \\
\text { plutonium }\end{array}\end{array}$} \\
\hline 8. Plutonium-239/240 & 1 & 33.9 & $N V^{c}$ & $N V^{c}$ & \\
\hline 9. Strontium-90 & 1 & 4.5 & $N V^{c}$ & $N V^{c}$ & $\begin{array}{l}\text { Gas flow } \\
\text { proportional } \\
\text { counting }\end{array}$ \\
\hline 10. Uranium-233/234 & 1 & $1.1^{\mathrm{d}}$ & $1.1^{\mathrm{d}}$ & $1.1^{\mathrm{d}}$ & \multirow{3}{*}{$\begin{array}{l}\text { 4. Isotopic - } \\
\text { uranium }\end{array}$} \\
\hline 11. Uranium-235 & 1 & 0.61 & $0.185^{\mathrm{e}}$ & $0.185^{\mathrm{e}}$ & \\
\hline 12. Uranium-238 & 1 & $1.1^{\mathrm{d}}$ & $1.1^{\mathrm{d}}$ & $1.1^{\mathrm{d}}$ & \\
\hline 13. Carbon-14 & 2 & 5.16 & $\mathrm{NV}^{\mathrm{c}}$ & $\mathrm{NV}^{\mathrm{c}}$ & \multirow{4}{*}{$\begin{array}{l}\text { 5. Liquid } \\
\text { scintillation } \\
\text { counter }\end{array}$} \\
\hline 14. Nickel-63 & 30 & 4,026 & $\mathrm{NV}^{\mathrm{c}}$ & $\mathrm{NV}^{\mathrm{c}}$ & \\
\hline 15. Tritium & 10 & 510 & 15.8 & 15.8 & \\
\hline 16. Technetium-99 & 0.25 & 5.7 & 0.46 & 0.46 & \\
\hline \multicolumn{6}{|c|}{ Nonradionuclides } \\
\hline 17. Antimony & 6 & 32 & $5.4^{\mathrm{e}}$ & 25.3 & \multirow{9}{*}{$\begin{array}{l}\text { 6. EPA } 6010 \text { (ICP } \\
\text { metal) }\end{array}$} \\
\hline 18. Aluminum & 5 & 80,000 & 480,000 & 960,000 & \\
\hline 19. Arsenic & 10 & $20^{f}$ & $20^{f}$ & $20^{f}$ & \\
\hline 20. Barium & 2 & 16,000 & 1,650 & 3,300 & \\
\hline 21. Beryllium & 0.5 & 160 & 63.2 & 126 & \\
\hline 22. Cadmium & 0.5 & 80 & 0.69 & $0.25^{\mathrm{e}}$ & \\
\hline 23. Chromium (total) & 1 & 120,000 & 2,000 & 2,600 & \\
\hline 24. Cobalt & 2 & 24 & $15.7^{d}$ & $N V^{c}$ & \\
\hline 25. Copper & 1 & 3,200 & 284 & 1,150 & \\
\hline 26. Lead & 5 & 250 & 3,000 & 840 & \multirow{8}{*}{$\begin{array}{l}\text { 7. EPA } 6010 \text { (ICP } \\
\text { metal) }\end{array}$} \\
\hline 27. Manganese & 5 & 3,760 & $512^{d}$ & $512^{d}$ & \\
\hline 28. Nickel & 4 & 1,600 & 130 & 357 & \\
\hline 29. Selenium & 10 & 400 & $5.2^{e}$ & $1.04^{\mathrm{e}}$ & \\
\hline 30. Silver & 1 & 400 & 13.6 & $0.884^{\mathrm{e}}$ & \\
\hline 31. Thallium & 5 & 5.60 & $1.59^{e}$ & $4.46^{\mathrm{e}}$ & \\
\hline 32. Vanadium & 2.5 & 560 & 2,240 & $\mathrm{NV}^{\mathrm{c}}$ & \\
\hline 33. Zinc & 1 & 24,000 & 5,970 & 226 & \\
\hline
\end{tabular}


Table 4. Master 100-K Target Analyte List. (3 Pages)

\begin{tabular}{|c|c|c|c|c|c|}
\hline \multirow[b]{2}{*}{ Target Analyte } & \multirow{2}{*}{$\begin{array}{c}\text { Practical } \\
\text { Quantitation }^{\text {Limits }}{ }^{\mathrm{a}}\end{array}$} & \multicolumn{3}{|c|}{ Preliminary Cleanup Goals ${ }^{a, b}$} & \multirow[b]{2}{*}{ Analytical Methods } \\
\hline & & $\begin{array}{c}\text { Direct } \\
\text { Exposure }\end{array}$ & $\begin{array}{c}\text { Groundwater } \\
\text { Protection }\end{array}$ & $\begin{array}{c}\text { River } \\
\text { Protection }\end{array}$ & \\
\hline 34. Chromium (hexavalent) & 0.5 & 240 & 18.4 & 7.7 & 8. Cr VI 7196 \\
\hline 35. Mercury & 0.2 & 24 & 2.09 & $0.33^{d}$ & $\begin{array}{l}\text { 9. EPA } 7471(\mathrm{Hg} \\
\text { cold vapor) }\end{array}$ \\
\hline 36. Aroclor-1016 (PCB) & 0.017 & 0.5 & 0.0942 & $0.000447^{\mathrm{e}}$ & \multirow{7}{*}{$\begin{array}{l}\text { 10. EPA } 8082 \text { (PCB } \\
\text { by GC) }\end{array}$} \\
\hline 37. Aroclor-1221 (PCB) & 0.017 & 0.5 & $0.00920^{\mathrm{e}}$ & $0.0000437^{\mathrm{e}}$ & \\
\hline 38. Aroclor-1232 (PCB) & 0.017 & 0.5 & $0.00920^{\mathrm{e}}$ & $0.0000437^{\mathrm{e}}$ & \\
\hline 39. Aroclor-1242 (PCB) & 0.017 & 0.5 & 0.0394 & $0.000187^{\mathrm{e}}$ & \\
\hline 40. Aroclor-1248 (PCB) & 0.017 & 0.5 & 0.0386 & $0.000183^{\mathrm{e}}$ & \\
\hline 41. Aroclor-1254 (PCB) & 0.017 & 0.5 & 0.0664 & $0.000315^{\mathrm{e}}$ & \\
\hline 42. Aroclor-1260 (PCB) & 0.017 & 0.5 & 0.721 & $0.00342^{\mathrm{e}}$ & \\
\hline 43. Fluoride & 5 & 4,800 & 2,880 & 5,770 & \multirow{3}{*}{$\begin{array}{l}\text { 11. Anions by } \\
\text { IC- } 300.0\end{array}$} \\
\hline 44. Nitrate & 2.5 & 128,000 & 40 & 80 & \\
\hline 45. Nitrite & 2.5 & 8,000 & 4 & 8 & \\
\hline 46. Acenaphthene & 0.1 & 4,800 & 97.9 & 131 & \multirow{10}{*}{ 12. EPA-8310 (PAH) } \\
\hline 47. Anthracene & 0.05 & 24,000 & 2,270 & 9,100 & \\
\hline 48. Benzo(a)anthracene & 0.015 & 1.37 & 0.856 & 0.04 & \\
\hline 49. Benzo(a)pyrene & 0.015 & 0.137 & 2.33 & 0.109 & \\
\hline 50. Benzo(b) fluoranthene & 0.015 & 1.37 & 2.95 & 0.138 & \\
\hline 51. Benzo(ghi) perylene & 0.03 & 2,400 & 25,700 & 7,070 & \\
\hline 52. Benzo(k) fluoranthene & 0.015 & 1.37 & 21.5 & 0.138 & \\
\hline 53. Chrysene & 0.1 & 13.7 & 9.56 & $0.0446^{\mathrm{e}}$ & \\
\hline 54. Dibenz $(\mathrm{a}, \mathrm{h})$ anthracene & 0.03 & 1.37 & 4.29 & 0.2 & \\
\hline 55. Fluoranthene & 0.05 & 3,200 & 631 & 178 & \\
\hline $\begin{array}{l}\text { 56. Indeno (1,2,3-cd) } \\
\text { pyrene }\end{array}$ & 0.03 & 1.37 & 8.33 & 0.389 & \multirow{3}{*}{ 13. EPA-8310 (PAH) } \\
\hline 57. Phenanthrene & 0.05 & 24,000 & 1,140 & 9,100 & \\
\hline 58. Pyrene & 0.05 & 2,400 & 655 & 2,620 & \\
\hline 59. Ethylene glycol & 5 & 160,000 & 64.3 & $N V^{c}$ & $\begin{array}{l}\text { 14. EPA-8015 (Non- } \\
\text { halogenated } \\
\text { VOA) }\end{array}$ \\
\hline 60. 1,1-Dichloroethene & 0.01 & 1.67 & 0.0005 & 0.0008 & \multirow{5}{*}{$\begin{array}{l}\text { 15. EPA-8270 } \\
\text { (Semi-volatiles) }\end{array}$} \\
\hline $\begin{array}{l}\text { 61. Bis (2-ethylhexyl) } \\
\text { phthalate }\end{array}$ & 0.33 & 71.4 & 13.9 & 8.01 & \\
\hline 62. Carbazole & 0.33 & 50 & $0.314^{\mathrm{e}}$ & $N V^{c}$ & \\
\hline 63. Di-n-butylphthalate & 0.33 & 8,000 & 56.5 & 191 & \\
\hline 64. Pentachlorophenol & 0.33 & 8.33 & $0.0158^{\mathrm{e}}$ & $0.00887^{\mathrm{e}}$ & \\
\hline 65. Benzene ${ }^{9}$ & 0.005 & 18.2 & 0.00448 & 0.014 & \multirow{8}{*}{$\begin{array}{l}\text { 16. EPA-8260 } \\
\text { (Volatile } \\
\text { organics) }\end{array}$} \\
\hline 66. Carbon tetrachloride & 0.005 & 7.69 & 0.031 & $0.0046^{\mathrm{e}}$ & \\
\hline 67. Chloroform & 0.005 & 164 & 0.038 & 0.0607 & \\
\hline 68. 1,4-Dichlorobenzene & 0.005 & 41.7 & 0.03 & 0.160 & \\
\hline 69. Methylene chloride & 0.005 & 133 & 0.0218 & 0.0409 & \\
\hline 70. Tetrachloroethene & 0.005 & 1.85 & 0.00859 & 0.00832 & \\
\hline 71. Toluene & 0.005 & 6,400 & 4.65 & 99 & \\
\hline 72. Trichloroethylene & 0.005 & 11.2 & $0.00323^{\mathrm{e}}$ & 0.0355 & \\
\hline
\end{tabular}


Table 4. Master 100-K Target Analyte List. (3 Pages)

\begin{tabular}{|c|c|c|c|c|c|}
\hline \multirow[b]{2}{*}{ Target Analyte } & \multirow{2}{*}{$\begin{array}{c}\text { Practical } \\
\text { Quantitation }_{\text {Limits }^{a}} \\
\end{array}$} & \multicolumn{3}{|c|}{ Preliminary Cleanup Goals $^{a, b}$} & \multirow[b]{2}{*}{ Analytical Methods } \\
\hline & & $\begin{array}{c}\text { Direct } \\
\text { Exposure }\end{array}$ & $\begin{array}{l}\text { Groundwater } \\
\text { Protection }\end{array}$ & $\begin{array}{c}\text { River } \\
\text { Protection }\end{array}$ & \\
\hline $\begin{array}{l}\text { 73. Total petroleum } \\
\text { hydrocarbons }\end{array}$ & 5 & 2,000 & 2,000 & $N V^{c}$ & $\begin{array}{l}\text { 17. NWTPH-Dx, } \\
\text { extended range }\end{array}$ \\
\hline
\end{tabular}

a Units are $\mathrm{mg} / \mathrm{kg}$ (nonradionuclides) and $\mathrm{pCi} / \mathrm{g}$ (radionuclides) unless otherwise noted.

b The initial cleanup goals presented here are from the most current CLARC table (updated April 22, 2009), calculated per Model Toxics Control Act Statute and Regulation, Publication No. 94-06, Washington State Department of Ecology, Olympia, Washington (WAC 173-340) (Ecology 2007) using input parameters stated in the CLARC table.

c The generic RESRAD modeling reported in the Remedial Design Report/Remedial Action Work Plan for the 100 Area (100 Area RDR/RAWP) (DOE/RL-96-17) predicts the contaminant will not reach groundwater within 1,000 years.

${ }^{d}$ Where cleanup levels are less than background, cleanup levels default to background as discussed in Section 2.1.2.1 of the 100 Area RDR/RAWP (DOE-RL-96-17).

e Where cleanup levels are less than PQLs, cleanup levels default to PQLs as discussed in Sec. 2.1.2.1 of the 100 Area RDR/RAWP (DOE/RL-96-17). The PQLs will be used for working levels, and will be periodically reviewed to establish if lower detection limit capabilities have become available.

$\mathrm{f}$ The arsenic cleanup level of $20 \mathrm{mg} / \mathrm{kg}$ has been agreed to by the Tri-Party Agreement project managers as discussed in Sec. 2.1.2.1 of the 100 Area RDR/RAWP (DOE/RL-96-17).

$\begin{array}{llll}\text { CLARC } & =\text { Cleanup Levels and Risk Calculation } & \text { PAH } & =\text { polycyclic aromatic hydrocarbons } \\ \text { COPC } & =\text { contaminant of potential concern } & \text { PCB } & =\text { polychlorinated biphenyl } \\ \text { EPA } & =\text { U.S. Environmental Protection Agency } & \text { PQL } & =\text { practical quantitation limit } \\ \text { GC } & =\text { gas chromatograph } & \text { RDR/RAWP } & =\text { Remedial Design Report/Remedial Action Work Plan } \\ \text { IC } & =\text { ion chromatography } & \text { RESRAD } & =\text { RESidual RADioactivity } \\ \text { ICP } & =\text { inductively coupled plasma } & \text { VOA } & =\text { volatile organic analysis } \\ \text { NV } & =\text { no value } & \text { WAC } & =\text { Washington Administrative Code }\end{array}$

NWTPH-Dx = Northwest total petroleum hydrocarbons-diesel range

\section{Location-Specific Target Analyte Identification}

Location-specific target analytes are identified from the master list using the following approach:

1. Identify the COCs for the specific waste sites where characterization is proposed from the applicable IAROD (which reflects information from LFI and technical baseline reports). If the characterization location is not at a waste site, evaluate information from waste sites in the vicinity (where available). Include these analytes on the location-specific target analyte list (Table 5).

2. Identify the COCs for the specific waste site locations from the verification documentation (CVPs or RSVPs). If the characterization location is not at a waste site, evaluate information from waste sites in the vicinity (where available). Include these analytes on the locationspecific target analyte list (Table 5).

3. Evaluate local groundwater monitoring well data (wells located within waste site "zones of influence"). Determine if groundwater COPCs have been analyzed for in these wells. The groundwater data for $100-\mathrm{K}$ is summarized in Appendix $\mathrm{A}$.

a. If the groundwater COPCs have been analyzed for but not detected, then these analytes will not be included on the location-specific target analyte list. 
b. If the groundwater COPCs have been analyzed for and have been detected, then these analytes are included on the location-specific target analyte list.

c. If the groundwater COPCs have not been analyzed for, then an additional evaluation will be performed to determine if there is a data need. If there is a data need, these COPCs are included on the location-specific target analyte list.

4. The analytes from the 100-K location-specific list were considered by the lead agency and no additional analytes were requested.

Table 5. 100-K Location-Specific Target Analyte List. (2 Pages)

\begin{tabular}{|c|c|c|c|c|c|}
\hline \multirow[b]{2}{*}{ Target Analyte } & \multirow{2}{*}{$\begin{array}{c}\text { Practical } \\
\text { Quantitation } \\
\text { Limits }^{\mathrm{a}}\end{array}$} & \multicolumn{3}{|c|}{ Preliminary Cleanup Goals $^{\mathrm{a}, \mathrm{b}}$} & \multirow[b]{2}{*}{$\begin{array}{l}\text { Analytical } \\
\text { Methods }\end{array}$} \\
\hline & & $\begin{array}{c}\text { Direct } \\
\text { Exposure }\end{array}$ & $\begin{array}{l}\text { Groundwater } \\
\text { Protection }\end{array}$ & $\begin{array}{c}\text { River } \\
\text { Protection }\end{array}$ & \\
\hline \multicolumn{6}{|c|}{ Radionuclides } \\
\hline 1. Cesium-137 (CVP) & 0.1 & 6.2 & $\mathrm{NV}^{\mathrm{C}}$ & $\mathrm{NV}^{\mathrm{C}}$ & \multirow{4}{*}{$\begin{array}{l}\text { 1. Gamma } \\
\text { energy } \\
\text { analysis }\end{array}$} \\
\hline 2. Co-60 (CVP/IAROD) & 0.05 & 1.4 & $\mathrm{NV}^{\mathrm{c}}$ & $\mathrm{NV}^{\mathrm{C}}$ & \\
\hline 3. Eu-152 (CVP/IAROD) & 0.1 & 3.3 & $N V^{c}$ & $N V^{c}$ & \\
\hline 4. Eu-154 (CVP/IAROD) & 0.1 & 3.0 & $\mathrm{NV}^{\mathrm{C}}$ & $\mathrm{NV}^{\mathrm{c}}$ & \\
\hline $\begin{array}{ll}\text { 5. } & \text { Pu-239/240 } \\
& \text { (CVP/IAROD) }\end{array}$ & 1 & 33.9 & $N V^{c}$ & $\mathrm{NV}^{\mathrm{c}}$ & 2. Isotopic-Pu \\
\hline 6. Sr-90 (CVP/IAROD) & 1 & 4.5 & $N V^{c}$ & $\mathrm{NV}^{\mathrm{c}}$ & $\begin{array}{l}\text { 3. Gas flow } \\
\text { proportional } \\
\text { counting }\end{array}$ \\
\hline 7. Carbon-14 (CVP) & 2 & 5.16 & $\mathrm{NV}^{\mathrm{C}}$ & $\mathrm{NV}^{\mathrm{C}}$ & \multirow{4}{*}{$\begin{array}{l}\text { 4. Liquid } \\
\text { scintillation } \\
\text { counter }\end{array}$} \\
\hline 8. Nickel-63 (CVP) & 30 & 4,026 & $N V^{c}$ & $\mathrm{NV}^{\mathrm{c}}$ & \\
\hline 9. Tritium (GW) & 10 & 510 & 15.8 & 15.8 & \\
\hline 10. Tc-99 (GW) & 0.25 & 5.7 & 0.46 & 0.46 & \\
\hline \multicolumn{6}{|c|}{ Nonradionuclides } \\
\hline 11. Antimony (GW) & 6 & 32 & $5.4^{\mathrm{d}}$ & 25.3 & \multirow{16}{*}{$\begin{array}{l}\text { 5. EPA } 6010 \\
\text { (ICP metal) }\end{array}$} \\
\hline 12. Arsenic (GW) & 10 & $20^{\mathrm{e}}$ & $20^{\mathrm{e}}$ & $20^{e}$ & \\
\hline 13. Barium (GW) & 2 & 16,000 & 1,650 & 3,300 & \\
\hline 14. Beryllium (GW) & 0.5 & 160 & 63.2 & 126 & \\
\hline 15. Cadmium (GW) & 0.5 & 80 & 0.69 & $0.25^{\mathrm{d}}$ & \\
\hline 16. Chromium (total) (GW) & 1 & 120,000 & 2,000 & 2,600 & \\
\hline 17. Cobalt (GW) & 2 & 24 & $15.7^{\dagger}$ & $N V^{c}$ & \\
\hline 18. Copper (GW) & 1 & 3,200 & 284 & 1,150 & \\
\hline 19. Lead (GW) & 5 & 250 & 3,000 & 840 & \\
\hline 20. Manganese (GW) & 5 & 3,760 & $512^{\dagger}$ & $512^{\dagger}$ & \\
\hline 21. Nickel (GW) & 4 & 1,600 & 130 & 357 & \\
\hline 22. Selenium (GW) & 10 & 400 & $5.2^{\mathrm{d}}$ & $1.04^{\mathrm{d}}$ & \\
\hline 23. Silver (GW) & 1 & 400 & 13.6 & $0.884^{\mathrm{d}}$ & \\
\hline 24. Thallium (GW) & 5 & 5.60 & $1.59^{d}$ & $4.46^{\mathrm{d}}$ & \\
\hline 25. Vanadium (GW) & 2.5 & 560 & 2,240 & $\mathrm{NV}^{\mathrm{c}}$ & \\
\hline 26. Zinc (GW) & 1 & 24,000 & 5,970 & 226 & \\
\hline $\begin{array}{l}\text { 27. Chromium } \\
\text { (hexavalent) } \\
\text { (CVP/IAROD) }\end{array}$ & 0.5 & 240 & 18.4 & 7.7 & 6. Cr-VI 7196 \\
\hline
\end{tabular}


Table 5. 100-K Location-Specific Target Analyte List. (2 Pages)

\begin{tabular}{|c|c|c|c|c|c|}
\hline \multirow[b]{2}{*}{ Target Analyte } & \multirow{2}{*}{$\begin{array}{c}\text { Practical } \\
\text { Quantitation } \\
\text { Limits }^{a}\end{array}$} & \multicolumn{3}{|c|}{ Preliminary Cleanup Goals ${ }^{a, b}$} & \multirow[b]{2}{*}{$\begin{array}{l}\text { Analytical } \\
\text { Methods }\end{array}$} \\
\hline & & $\begin{array}{c}\text { Direct } \\
\text { Exposure }\end{array}$ & $\begin{array}{c}\text { Groundwater } \\
\text { Protection }\end{array}$ & $\begin{array}{c}\text { River } \\
\text { Protection }\end{array}$ & \\
\hline 28. Mercury (GW/IAROD) & 0.2 & 24 & 2.09 & $0.33^{f}$ & $\begin{array}{l}\text { 7. } \begin{array}{l}\text { EPA } 7471 \\
\text { (Hg cold } \\
\text { vapor) }\end{array} \\
\end{array}$ \\
\hline 29. Fluoride (GW) & 5 & 4,800 & 2,880 & 5,770 & \multirow{3}{*}{$\begin{array}{l}\text { 8. Anions by } \\
\text { IC-300.0 }\end{array}$} \\
\hline 30. Nitrate (GW) & 2.5 & 128,000 & 40 & 80 & \\
\hline 31. Nitrite (GW) & 2.5 & 8,000 & 4 & 8 & \\
\hline 32. Benzene (GW) & 0.005 & 18.2 & $0.00448^{d}$ & 0.014 & \multirow{2}{*}{\begin{tabular}{|ll} 
9. & EPA-8260 \\
(Volatile \\
organics)
\end{tabular}} \\
\hline 33. Chloroform (GW) & 0.005 & 164 & 0.0381 & 0.0607 & \\
\hline
\end{tabular}

${ }^{\text {a }}$ Units are $\mathrm{mg} / \mathrm{kg}$ (nonradionuclides) and $\mathrm{pCi} / \mathrm{g}$ (radionuclides) unless otherwise noted.

b The initial cleanup goals presented here are from the most current CLARC table (updated April 22, 2009), calculated per Model Toxics Control Act Statute and Regulation, Publication No. 94-06, Washington State Department of Ecology, Olympia, Washington (WAC 173-340) (Ecology 2007) using input parameters stated in the CLARC table.

c The generic RESRAD modeling reported in the Remedial Design Report/Remedial Action Work Plan for the 100 Area (100 Area RDR/RAWP) (DOE/RL-96-17) predicts the contaminant will not reach groundwater within 1,000 years.

${ }^{d}$ Where cleanup levels are less than PQLs, cleanup levels default to PQLs as discussed in Sec. 2.1.2.1 of the 100 Area RDR/RAWP (DOE/RL-96-17). The PQLs will be used for working levels, and will be periodically reviewed to establish if lower detection limit capabilities have become available.

e The arsenic cleanup level of $20 \mathrm{mg} / \mathrm{kg}$ has been agreed to by the Tri-Party Agreement project managers as discussed in Sec. 2.1.2.1 of the 100 Area RDR/RAWP (DOE/RL-96-17).

$f$ Where cleanup levels are less than background, cleanup levels default to background as discussed in Sec. 2.1.2.1 of the 100 Area RDR/RAWP (DOE/RL-96-17).

$\begin{array}{llll}\text { CLARC } & =\text { Cleanup Levels and Risk Calculation } & \text { ICP } & =\text { inductively coupled plasma } \\ \text { CVP } & =\text { cleanup verification package } & \text { NV } & =\text { no value } \\ \text { EPA } & =\text { U. S. Environmental Protection Agency } & \text { PQL } & =\text { practical quantitation limit } \\ \text { GW } & \text { groundwater } & \text { RDR/RAWP } & =\text { Remedial Design Report/Remedial Action Work Plan } \\ \text { IAROD } & =\text { interim action record of decision } & \text { RESRAD } & =\text { RESidual RADioactivity } \\ \text { IC } & =\text { ion exchange chromatography } & \text { WAC } & =\text { Washington Administrative Code }\end{array}$

\subsection{CONCLUSIONS}

This approach should be followed to identify target analytes for the other 100 and 300 Area RI/FS work plans under development.

The analytical methods in Tables 4 and 5, particularly those identified for radionuclides, should be verified and documented in the quality assurance project plan section of the sampling and analysis plan for the 100-K Area. As additional soil data become available, other suitable exclusion criteria should be considered and evaluated for use in the target analyte list development process. 


\subsection{REFERENCES}

2003-036, 2004, Waste Site Reclassification Form 2003-036, and Attachment Remaining Sites Verification Package for 100-K-30, 183-KE Sulfuric Acid Tank (West Tank), Rev. 0, Washington Closure Hanford, Richland, Washington.

2004-038, 2004, Waste Site Reclassification Form 2004-038, and Attachment Remaining Sites Verification Package for 100-K-31, 183-KE Sulfuric Acid Tank (East Tank), Rev. 0, Washington Closure Hanford, Richland, Washington.

2004-039, 2004, Waste Site Reclassification Form 2004-039, and Attachment Remaining Sites Verification Package for 100-K-32, 183-KW Sulfuric Acid Tank Bases (East Tank), Rev. 0, Washington Closure Hanford, Richland, Washington.

2004-040, 2004, Waste Site Reclassification Form 2004-040, and Attachment Remaining Sites Verification Package for 100-K-29, 183-KE Sandblasting Site, Rev. 0, Washington Closure Hanford, Richland, Washington.

2004-041, 2004, Waste Site Reclassification Form 2004-041, and Attachment Remaining Sites Verification Package for 100-K-33, 183-KW Sulfuric Acid Tank Bases (West Tank), Rev. 0, Washington Closure Hanford, Richland, Washington.

2004-042, 2004, Waste Site Reclassification Form 2004-042, and Attachment Remaining Sites Verification Package for 128-K-1, 100-K Burning Pit, Rev. 0, Washington Closure Hanford, Richland, Washington.

BHI-01737, 2004, Cleanup Status Report for the 116-KE-1 and 116-KW-1 Cribs, Rev. 0, Bechtel Hanford, Inc., Richland, Washington.

CVP-2003-00024, 2003, Cleanup Verification Package for the 116-K-1 Crib, Rev. 0, Washington Closure Hanford, Richland, Washington.

CVP-2004-00001, 2004, Cleanup Verification Package for the 116-KW-3 Retention Basin, Rev. 0, Washington Closure Hanford, Richland, Washington.

CVP-2005-00002, 2005, Cleanup Verification Package for the 116-KE-4 Retention Basin, Rev. 0, Washington Closure Hanford, Richland, Washington.

CVP-2005-00006, 2005, Cleanup Verification Package for the 100-K-55:1 and 100-K-56:1 Pipelines and the 116-KW-4 and 116-KE-5 Heat Recovery Stations, Rev. 0, Washington Closure Hanford, Richland, Washington.

CVP-2006-00001, 2006, Cleanup Verification Package for the 116-K-2 Effluent Trench, Rev. 0, Washington Closure Hanford, Richland, Washington.

DOE/RL-93-78, 1994, Limited Field Investigation Report for the 100-KR-1 Operable Unit, Rev. 0, U.S. Department of Energy, Richland Operations Office, Richland, Washington.

DOE/RL-94-61,1995, 100 Area Source Operable Unit Focused Feasibility Study Report, Rev. 0, U.S. Department of Energy, Richland Operations Office, Richland, Washington.

DOE/RL-96-17, 2009, Remedial Design Report/Remedial Action Work Plan for the 100 Area, Rev. 6, U.S. Department of Energy, Richland Operations Office, Richland, Washington. 
DOE/RL-2008-33, 2008, Sampling and Analysis Plan for Investigating Chromium Groundwater Contamination Near the 105-KW Reactor, Rev. 0, U.S. Department of Energy, Richland Operations Office, Richland, Washington.

DOE/RL-2008-46, 2010, Integrated 100 Area Remedial Investigation/Feasibility Study Work Plan, Rev. 0, U.S. Department of Energy, Richland Operations Office, Richland, Washington.

Dorian, J. J. and V. R. Richards, 1978, Radiological Characterization of the Retired 100 Areas, UNI-946, United Nuclear Industries, Inc., Richland, Washington.

Ecology, 1996, "Model Toxics Control Act - Cleanup," Washington Administrative Code (WAC) 173-340, Washington State Department of Ecology, Olympia, Washington.

Ecology, 2007, Model Toxics Control Act Statute and Regulation, Publication No. 94-06, Washington State Department of Ecology, Olympia, Washington.

Ecology, 2009, Cleanup Levels and Risk Calculations (CLARC) Database, Washington State Department of Ecology, Olympia, Washington, <https://fortress.wa.gov/ecy/clarc.CLARCHome.aspx>.

Ecology, 2009, Dangerous Waste Portion of the Resource Conservation and Recovery Act Permit for the Treatment, Storage, and Disposal of Dangerous Waste, Permit No. WA7 89000 8967, Closure Unit 16, 1706-KE Waste Treatment System, Washington State Department of Ecology, Olympia, Washington.

EPA/ROD/R10-00/121, 2000, Record of Decision for the 100-BC-1, 100-BC-2, 100-DR-1, 100-DR-2, 100-FR-2, 100-HR-2, and 100-KR-2 Operable Units, Hanford Site, Benton County, Washington, U.S. Environmental Protection Agency, Washington, D.C.

EPA/ROD/R10-99/039, 1999, Interim Action Record of Decision for the 100-BC-1, 100-BC-2, 100-DR-1, 100-DR-2, 100-FR-1, 100-FR-2, 100-HR-1, 100-HR-2, 100-KR-1, 100-KR-2, 100-IU-2, 100-IU-6, and 200-CW-3 Operable Units, Hanford Site, Benton County, Washington, U.S. Environmental Protection Agency, Washington, D.C.

EPA/ROD/R10-99/059, 1999, Record of Decision for the 100-KR-2 Operable Unit, Hanford Site, Benton County, Washington, U.S. Environmental Protection Agency, Washington, D.C.

OSWER Directive 9285.7-53, 2003, Human Health Toxicity Values in Superfund Risk Assessments, to Superfund National Policy Managers, Regions 1-10, from M.B. Cook (Director, Office of Superfund Remediation and Technology Innovation), U.S. Environmental Protection Agency, Office of Solid Waste and Emergency Response, Washington, D.C., December 5.

WA7 89000 8967, 2009, Dangerous Waste Portion of the Resource Conservation and Recovery Act Permit for the Treatment, Storage, and Disposal of Dangerous Waste, Permit No. WA7 89000 8967, Closure Unit 16, 1706-KE Waste Treatment System, Washington State Department of Ecology, Olympia, Washington. 
APPENDIX A

100-K TARGET ANALYTE MEASLES CHART 
Table A-1. 100-K Target Analyte Measles Chart. (2 Pages)

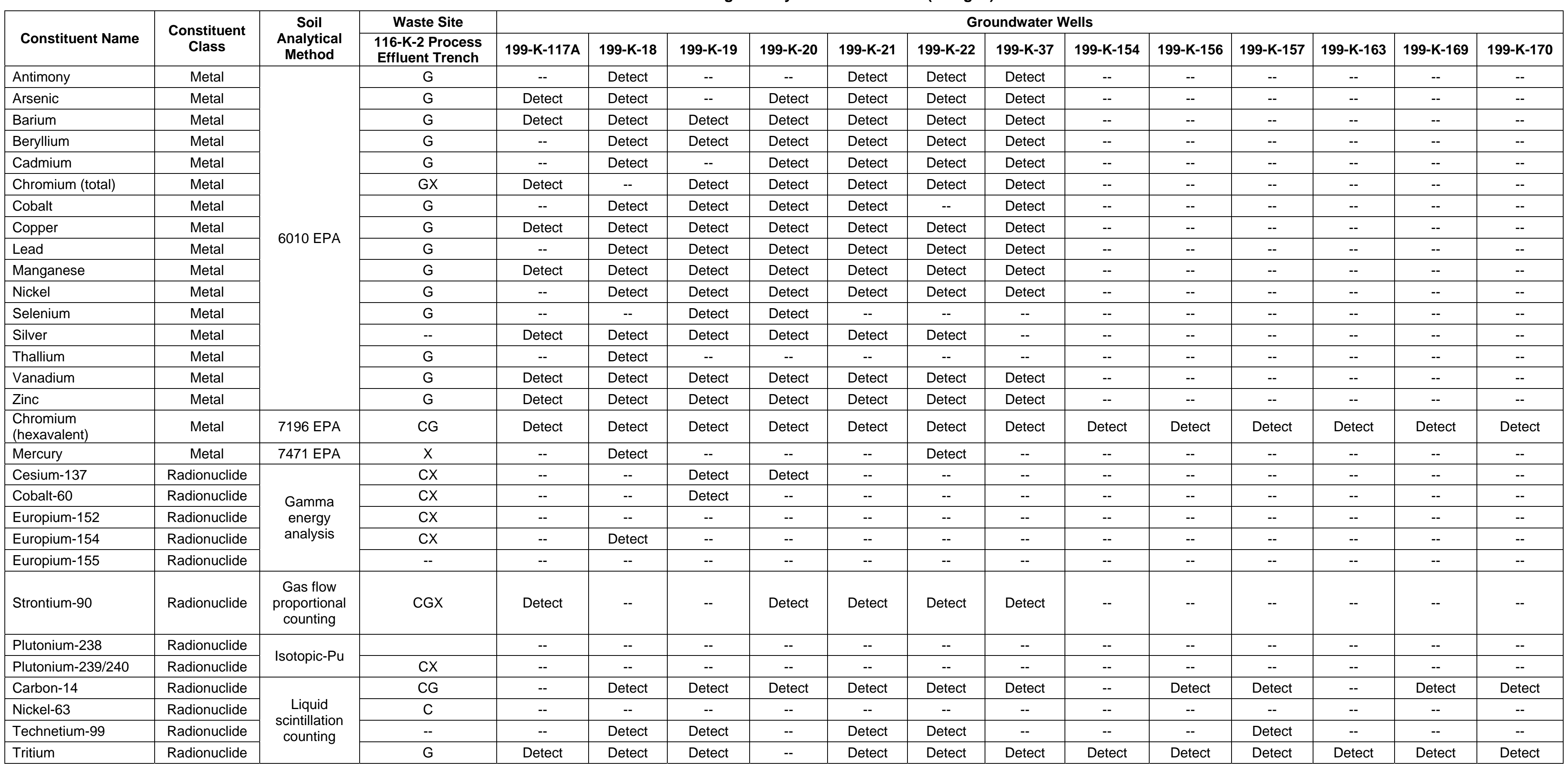


Table A-1. 100-K Target Analyte Measles Chart. (2 Pages)

\begin{tabular}{|c|c|c|c|c|c|c|c|c|c|c|c|c|c|c|c|c|}
\hline \multirow[b]{2}{*}{ Constituent Name } & \multirow{2}{*}{$\begin{array}{l}\text { Constituent } \\
\text { Class }\end{array}$} & \multirow{2}{*}{$\begin{array}{c}\text { Soil } \\
\text { Analytical } \\
\text { Method }\end{array}$} & \multirow{2}{*}{$\begin{array}{c}\text { Waste Site } \\
\text { 116-K-2 Process } \\
\text { Effluent Trench }\end{array}$} & \multicolumn{13}{|c|}{ Groundwater Wells } \\
\hline & & & & 199-K-117A & 199-K-18 & 199-K-19 & 199-K-20 & 199-K-21 & 199-K-22 & 199-K-37 & 199-K-154 & 199-K-156 & 199-K-157 & 199-K-163 & 199-K-169 & 199-K-170 \\
\hline 1,1-Dichloroethene & VOC & \multirow{9}{*}{8260 EPA } & G & -- & -- & -- & -- & -- & -- & -- & -- & -- & -- & -- & -- & -- \\
\hline 1,1,2-Trichloroethane & VOC & & G & -- & -- & -- & -- & -- & -- & -- & -- & -- & -- & -- & -- & -- \\
\hline Benzene & VOC & & G & -- & Detect & -- & -- & Detect & Detect & -- & -- & -- & -- & -- & -- & -- \\
\hline Chloroform & VOC & & G & -- & Detect & Detect & Detect & Detect & Detect & Detect & -- & -- & -- & -- & -- & -- \\
\hline Carbon tetrachloride & VOC & & G & -- & -- & -- & -- & -- & -- & -- & -- & -- & -- & -- & -- & -- \\
\hline Tetrachloroethene & VOC & & G & -- & -- & -- & -- & -- & -- & -- & -- & -- & -- & -- & -- & -- \\
\hline Toluene & VOC & & -- & -- & -- & -- & -- & -- & -- & -- & -- & -- & -- & -- & -- & -- \\
\hline Trichloroethene & VOC & & G & -- & -- & -- & -- & -- & -- & -- & -- & -- & -- & -- & -- & -- \\
\hline Vinyl chloride & VOC & & G & -- & -- & -- & -- & -- & -- & -- & -- & -- & -- & -- & -- & -- \\
\hline Chloride & Wet chem & \multirow{5}{*}{9056 EPA } & G & Detect & Detect & Detect & Detect & -- & -- & -- & -- & -- & -- & -- & -- & -- \\
\hline Fluoride & Wet chem & & G & Detect & Detect & Detect & Detect & -- & -- & -- & -- & -- & -- & -- & -- & -- \\
\hline Nitrate $\left(\mathrm{NO}_{3}\right)$ & Wet chem & & G & Detect & Detect & Detect & Detect & Detect & Detect & Detect & -- & -- & -- & -- & -- & -- \\
\hline Nitrite $\left(\mathrm{NO}_{2}\right)$ & Wet chem & & G & Detect & Detect & Detect & -- & -- & -- & -- & -- & -- & -- & -- & -- & -- \\
\hline Sulfate & Wet chem & & G & Detect & Detect & Detect & Detect & Detect & Detect & Detect & -- & -- & -- & -- & -- & -- \\
\hline
\end{tabular}

$=$ analyte was either not analyzed in groundwater or not detected in groundwater

$\begin{aligned} C & =\text { identified in cleanup verification package } \\ \text { EPA } & =\text { U.S. Environmental Protection Agency }\end{aligned}$

$\mathrm{EPA}=$ = identified as a groundwater contaminant of potential concern

$\begin{aligned} \mathrm{VOC} & =\text { volatile organic compound } \\ \mathrm{X} & =\text { identified as an interim record of decision contaminant of concern }\end{aligned}$ 


\section{DISTRIBUTION}

U.S. Department of Energy

$\underline{\text { Richland Operations Office }}$

J. P. Hanson

Washington Closure Hanford

J. A. Lerch

K. M. Singleton

Document Control

DOE-RL Public Reading Room

Hanford Technical Library
A5-11

$\mathrm{H} 4-22$

$\mathrm{H} 4-22$

H4-11

$\mathrm{H} 2-53$

P8-55 